\title{
Foreword
}

\section{Can Artificial Intelligence Replace Human GO Players?}

\author{
Tokyo Metropolitan University, Masahiko HADA
}

I have loved playing the traditional oriental game of GO for the past forty-five years. In this game, we use a board with a grid pattern of $19 \times 19$, and players take turns and place the black and white stones on the grid points. I also have enjoyed the computer GO program. As opposed to other board games such as chess and shogi, computers have found it much more difficult to beat humans at $\mathrm{GO}$ because of huge number of branches involved. When IBM's computer Deep-Blue beat world chess Champion Garry Kasparov in 1997, the strongest computer GO program that used artificial intelligence (AI) techniques was only at level of amateur-5-dan. However, recent computer GO programs have developed beyond our expectations and are strong GO players. In 2016, global GO fans were surprised by the news that AlphaGO developed by Google-DeepMind beat the famous Korean professional GO player Lee Sedol. Moreover, in Japan, DeepZenGo, developed with support from Dowango, beat some famous top-level professional players. AlphaGo uses a Monte-Carlo tree search algorithm to find moves based on the knowledge previously acquired using machine learning. DeepZenGo also uses this algorithm as well as a value-network and policy-network with fine adjustments. The honorary championship GO player, Mr. Cho Chi Hun, was quoted saying, "We should learn good and novel moves devised by computer GO programs, especially in the early stage of the game, because human GO players use only feeling and experience then." In reality, the characteristic moves used by computer GO players, for example unexpected "ohgeima-shimari," have influenced some professional GO players, and these have also been observed in big title matches. Owing to the recent rise in computer GO programs, the conventional, standard formulae in GO seem to be turning and in some cases, have been phased out.

Has Human intelligence (HI) conceded defeat to AI in all cases such as GO? Recent advances have enabled computers to drive cars, pass high-level university entrance examinations, and accurately diagnose diseases in the human life. Computers can analyze large amounts of information accumulated using computer networks or machine-learning and output appropriate answers. Very recently, in many cases, HI has been surely and completely beaten by AI. In the near future, most queries will be answered by AI. This brings us to an inevitable question. What should we human beings do in the future? In what applications and cases will HI remain superior to AI? In my opinion, it is "to ask questions". Finding an answer itself is not as important, and therefore we can safely entrust the task of "finding answers" to diligent computers. However, what constitutes a worthwhile question? Only this point remains a task that HI can complete. Maybe someday in the future, AI can answer the Buddha's famous question "How can we overcome the four uncontrollable phenomena, survival, disease, aging, and death?". 